\title{
PREPARATION OF OXYGEN OF HIGH PURITY
}

\author{
By Martin Shepherd, E. R. Weaver, and S. F. Pickering
}

\section{ABSTRAC'T}

Oxygen, which contained no significant amounts of lower- or higher-boiling impurities, was prepared by a method whose essential feature was the elimination of nitrogen. This oxygen is one of several reference samples being used in connection with the maintenance of the International Temperature Scale at the National Bureau of Standards.

\section{CONTENTS}

I. Essential feature of the method .

II. Apparatus .

III. Procedure _. 303

1. Removal of dissolved gases from the electrolyte $\ldots \ldots . \ldots 303$

2. Removal of adsorbed and occluded gases from the platinum

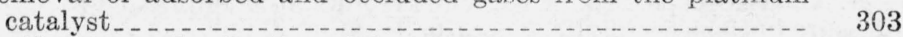

3. Purification and collection of the oxygen $\ldots \ldots \ldots \ldots$

IV. Purity of the oxygen

V. Significance of the impurities

VI. Final treatment and transfer of oxygen to the saturation pressure thermometer

\section{ESSENTIAL FEATURE OF THE METHOD}

Oxygen of high purity has been prepared by various chemical and physical methods. A complete bibliography of the subject would exceed the length of this paper. ${ }^{1}$ Two important facts to be derived from a study of the literature are: (1) the methods of preparation did not exclude the possibility of traces of nitrogen as an impurity, and (2) the methods of purification may have introduced additional traces of nitrogen. Distillation, sometimes resorted to for the final purification, probably failed to remove the last traces of nitrogen.

These facts become apparent when the methods of preparation and purification are considered. Some nitrogen may be expected in oxygen prepared from the electrolysis, thermal dissociation, or reaction of solid or liquid reagents containing dissolved or occluded nitrogen. Subsequently, oxygen purified by passage through solid or liquid reagents will become contaminated with nitrogen dissolved or occluded in these reagents. The complete removal of nitrogen from such reagents, prior to the preparation and purification of oxygen, requires more exacting procedures than have been employed previously.

1 For representative work of the most exacting nature, the following references are given: Morley, Smithsonian Contributions to Knowledge (1895); Germann, J. Phys. Chem. 19, 437 (1915); Moles and Gonzales, J. chim. phys. 19, 310 (1921); and Baxter and Starkweather, Proc. Nat. Acad. Sci. U. S. 19, 481 (1924). 
Finally, in oxygen separated from air, nitrogen (and argon) are the predominant impurities to be expected. Distillation in the laboratory can scarcely be expected to remove these impurities completely. ${ }^{2}$ Similarly, distillation cannot be trusted to remove the last traces of nitrogen from oxygen prepared chemically.

The essential feature of the method herein described is the critical removal of nitrogen from both reagents and apparatus prior to the actual generation and purification of the oxygen. With this primary consideration in mind, the electrolysis of an aqueous solution of barium hydroxide was selected as the most suitable means of producing oxygen. There are good reasons for this choice. Dissolved gases may be completely removed from the electrolyte before the oxygen is prepared. Thereafter, no impurities are introduced which cannot be removed by combustion over heated platinum and subsequent condensation to negligible vapor pressures. Occluded gases can be removed from the platinum catalyst before the combustion. Necessary reagents are reduced to a minimum, and no extensive purification "train" is involved. Solid reagents, which are most apt to introduce nitrogen, are avoided with the single exception of platinum.

\section{APPARATUS}

The photograph of the apparatus is lettered in alphabetical sequence for the reader's convenience. The various parts are:

$A$, vacuum-type needle valve with fine-pitch adjustment for controlling the pressure in the electrolytic generator.

$B$, barometric mercury seal serving as a manometer and pressure relief.

$C$, condenser.

$D$, electrolytic cell. The platinum electrodes are sealed through glass tubes entering at the top. These tubes are filled with mercury to provide contact with the source of power. Glass-wool filters at the exits prevent the entrainment of spray.

$E$, condenser.

$F$, barometric mercury seal.

$G$, combustion tube. This is made of Pyrex glass, and is a water-jacketed tube of 5 -mm bore which contains a platinum spiral $300 \mathrm{~mm}$ in length. It is provided with a small condenser at the outlet. Such tubes have been described by Weaver and Ledig. ${ }^{3}$

$H$, condenser with glass-wool filter.

$I$, McLeod gage for measuring vacuum.

$J$, condenser and distillation tube with glass-wool filter.

$K$, graduated capillary for measuring small amounts of liquid oxygen.

$L$, saturation pressure thermometer to be filled with oxygen.

$M$, condenser.

$N$, differential manometer used in measuring the purity of the oxygen.

$O$, barometric mercury seal.

$P$, two-stage mercury-vapor pump (backed by a Hyvac pump, not shown).

$X$, a bypass, not shown in the photograph, is sealed to the two horizontal exit tubes of the generator, $D$. This bypass consists of an inverted $U$ connection with a stopcock.

The stopcocks of the apparatus are conveniently numbered. They are one-way, oblique-bored cocks of the hollow-blown type, with especial grinding suitable for vacuum work.

The apparatus is fused together as a single unit. (The four rubber tubes shown in the photograph carry cooling water to and from the jackets of the combustion tube and pump.) The portion to the left

2 Exhaustive fractional rectification of large amounts of oxygen might possibly accomplish this desideratum, but the literature affords no evidence of efforts which were sufficiently heroic.

${ }^{3}$ E. R. Weaver and P. G. Ledig, J. Ind. Eng. Chem. 12, 368 (1920). 


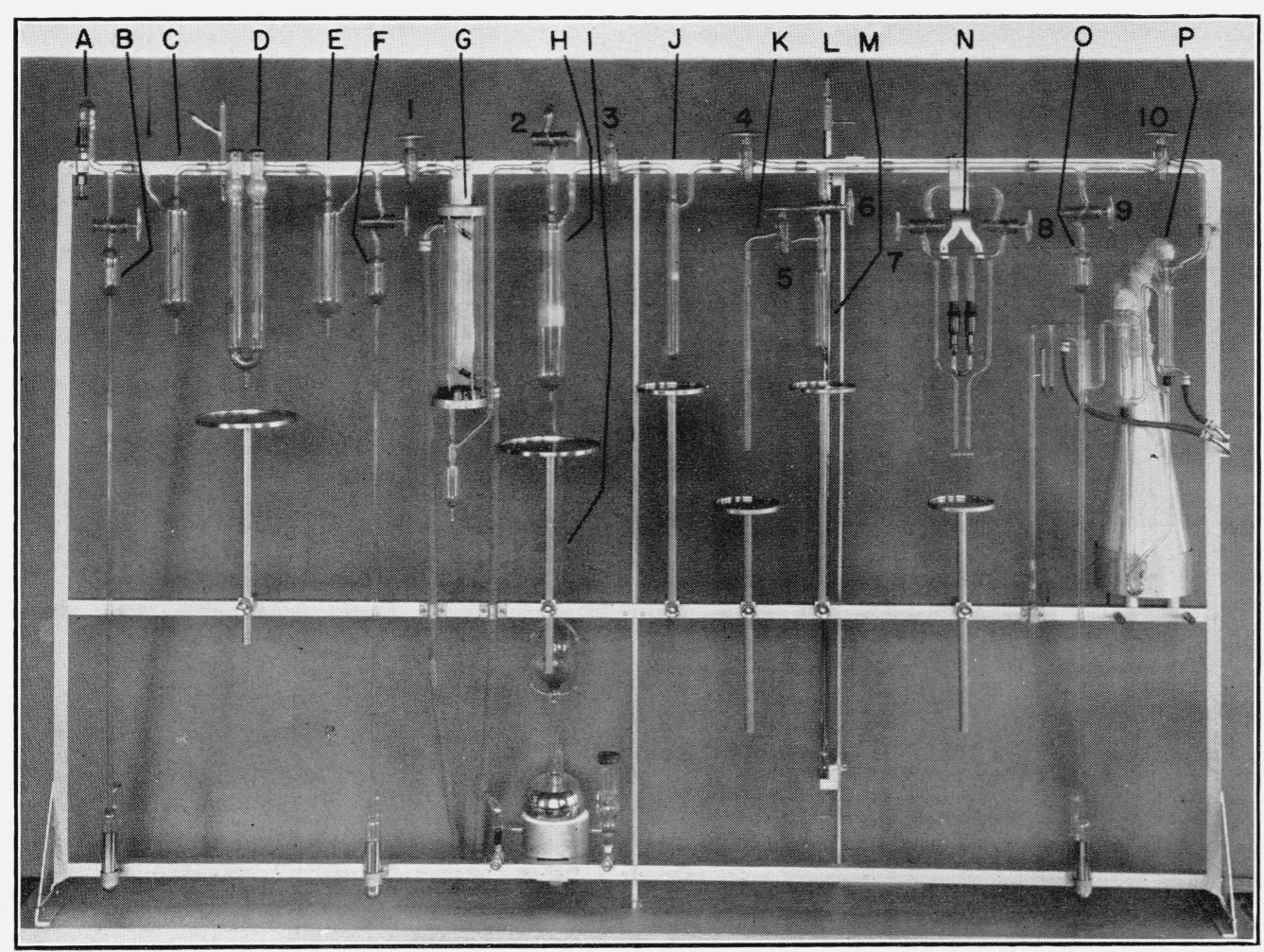

FIGURE 1.-A pparatus for preparing oxygen of high purity. 
of the combustion tube is blown from a soda-lime glass, and the rest is made of Pyrex glass. A graded glass seal serves to join the two sections.

\section{PROCEDURE}

\section{REMOVAL OF DISSOLVED GASES FROM THE ELECTROLYTE}

Barium hydroxide of reagent quality was dissolved in distilled water, and the filtered solution was transferred to the electrolytic cell through the top inlet, which was then fused shut. The solution was frozen within the cell, the entire apparatus was evacuated, and the condensers, $C$ and $E$, were immersed in liquid air. Ice from the cell was slowly sublimed into these condensers while the pump, $P$, was continuously withdrawing the liberated gases. Nearly all of the water was removed in this manner. The procedure was reversed to return the ice from $C$ and $E$ to the cell. This process of vacuum sublimation is known to be effective for removing gases from liquids. ${ }^{4}$

Following the vacuum sublimation of the electrolyte, oxygen and hydrogen were generated slowly over a period of about 100 hours. During this time, the bypass, $X$, was opened and stopcock 1 closed. The gases were withdrawn by a vacuum pump connected to the valve, A. A pressure slightly higher than the vapor pressure of the solution itself was maintained. Under this condition, the gases were evolved in such minute bubbles that the solution appeared milky. The bypass, $X$, was closed occasionally, and the solution in the cell was allowed to flow partially from one arm of the $U$ to the other, thus mixing the portion which lay below the electrodes. This whole procedure seems well suited to remove all dissolved gases except oxygen and hydrogen.

Thus two independent methods were used to critically remove dissolved gases from the solution of barium hydroxide.

\section{REMOVAL OF ADSORBED AND OCCLUDED GASES FROM THE PLATINUM CATALYST}

While the second of the two operations just described was being conducted, the platinum wire within the combustion tube was heated electrically to "whiteness," a temperature considerably in excess of that used subsequently for the combustion of hydrogen. The pump was connected to the combustion tube and to the entire right-hand section of the apparatus. When the pressure measured by the McLeod gage was reduced to $0.0001 \mathrm{~mm} \mathrm{Hg}$, the pump was disconnected by closing stopcock 9 . The pressure was observed to increase, which indicated that the hot platinum and glass reluctantly gave up the adsorbed and occluded gases. Evacuation was then resumed. When finally the pressure remained lower than $0.0001 \mathrm{~mm}$ for a period of 6 hours with the pump disconnected, the platinum was considered suitable for use as a catalyst. (The generation of the oxygen later collected required 6 hours.) This desired condition was achieved after about 120 hours of evacuation.

By the same criterion, the apparatus itself was judged to be sufficiently freed from adsorbed gases.

\footnotetext{
${ }^{4}$ One account of the process is given by J. H. Hibben, BS J. Research 3, 97 (1929) RP87.
} 


\section{PURIFICATION AND COLLECTION OF THE OXYGEN}

The by-pass, $X$, was now closed, and the valve, $A$, was adjusted so that the solution remained level in the two arms of the electrolytic cell. Since twice as much hydrogen as oxygen is generated and the cell itself is a sensitive manometer, the manipulation of the valve required considerable skill. The pressure in the cell was increased until hydrogen and oxygen bubbled through the seals, $B$ and $F$. Cocks 2 to 10 , inclusive, were closed. The condenser, $H$, was immersed in a bath of liquid oxygen. The temperature of the platinum wire was lowered from "white" to a "cherry red." Stopcock 1 was then opened very slowly, and the pressure was slowly increased in $G$ and $H$ to approximately $740 \mathrm{~mm}$. At this pressure and at the temperature of the bath of oxygen, the oxygen within the condenser did not liquefy. On the other hand, any higher-boiling impurities which might conceivably be present after the combustion were condensed to negligible vapor pressures. Any small frozen particles were retained on the glass-wool filter and thus could not escape to reappear upon vaporization in the effluent gas.

Following these operations, cocks 3, 4, and 9 were opened very slowly and in succession, and pressure was allowed to build up in each part of the apparatus so opened. Oxygen was then discarded through cock 10 until the apparatus was thoroughly purged. This precaution was observed because of unavoidable though very slight surges of pressure which occurred through the combustion tube during the period of adjustment of pressure throughout the apparatus.

The condenser, $J$, was now immersed in freshly liquefied air, and about $20 \mathrm{ml}$ of oxygen was condensed therein as fast as it was generated in $D$ and purified in $G$ and $H$. Thereafter, stopcock 3 was closed.

\section{PURITY OF THE OXYGEN}

The purity of the oxygen was measured by a sensitive method based on the observation of differential saturation pressures. ${ }^{5}$ The oxygen collected in the tube, $J$, was separated into three fractions by isothermal fractional distillation at $-200^{\circ} \mathrm{C}$. These fractions were: A very small amount of initial distillate; a middle fraction which was condensed in $M$; and a very small final residue. The saturation pressures of these fractions were compared by means of the differential manometer, $N$. During these measurements, the bulbs of the manometer which held the condensates were surrounded with a block of frozen mercury. After each comparative measurement, the positions of the condensates were interchanged to eliminate errors of capillarity.

The following observations were made:

1. No difference in pressure was observed when comparing the final residue with the middle fraction.

2. The saturation pressure of the initial distillate was $0.06 \mathrm{~mm}$ higher than that of the middle fraction.

3. The saturation pressure of the initial distillate was $0.06 \mathrm{~mm}$ higher than that of the final residue. Each of these observations represents the average of 10 determinations. These data indicate the absence of impurities of higher boiling points, and the presence of traces of impurities of lower boiling points.

\footnotetext{
${ }^{5}$ For complete details see, Martin Shepherd, BS J. Research 12, 185 (1934) RP643.
} 
The amount of any suspected impurity may be estimated from the equation $^{6}$

where

$$
X_{B}=\frac{\Delta_{p}}{p^{\circ}{ }_{B}-p^{\circ}{ }_{A}},
$$

$X_{B}=$ mole fraction of impurity present.

$\Delta p=$ differential saturation pressure observed with the manometer.

$p^{\circ}{ }_{B}$ and $p^{\circ}{ }_{A}=$ the saturation pressure of the impurity and the oxygen, each in the pure state.

If the impurity had been nitrogen, the data would account for the presence of 0.00003 mole fraction. The amount of nitrogen actually present would be slightly higher than indicated by this computed value. There is no reported temperature-composition diagram of the system nitrogen-oxygen under the conditions existing during the isothermal distillation employed in this test of purity; therefore, an exact calculation of the amount of nitrogen cannot be made. However, the isothermal distillation may be expected to isolate more of the nitrogen in the initial distillate than is possible by means of an isobaric distillation at $760 \mathrm{~mm}$. Furthermore, the data of Baly show only 2.2 mole percent of oxygen in the vapor phase at $78.0^{\circ} \mathrm{K}$ and $760 \mathrm{~mm}$. This at once indicates the high concentration of nitrogen which may be expected in the initial distillate, and it can be expected that the comparison of the saturation pressure of this distillate with that of the middle cut or final residue will produce almost the full effect which a nitrogen impurity would cause. This may also argue the efficacy of distillation as a means of final purification; but unfortunately such is not the usual case, for the elimination of the last traces of nitrogen by this means requires repeated fractionation and the wasting of large amounts of oxygen. Those who have previously employed this means of purification have reported no data which would definitely establish, or even give comforting assurance of, the purity of the product obtained.

If the impurity had been hydrogen, the amount detectable would be very much less than the estimated amount of nitrogen.

\section{SIGNIFICANCE OF THE IMPURITIES}

This oxygen was prepared for a reference sample in connection with the maintenance of the International Temperature Scale at the $\mathrm{Na}$ tional Bureau of Standards. The significance of the impurities detected, with particular respect to the measurement of saturation pressures, is accordingly important.

Since nitrogen and hydrogen are the only lower-boiling impurities to be expected, the effect of each should be calculated. The most unfavorable case to assume is that the impurities occuring in the initial distillate would also be present in the oxygen used to fill the saturation pressure thermometers. Actually, this was not the case, since the initial and final fractions were discarded; but if this assumption is made, the maximum possible error in the saturation pressures of oxygen would be $0.06 \mathrm{~mm} \mathrm{Hg}$, the $\Delta_{p}$ observed with the differential

\footnotetext{
- See eq 6 on page 191 of BS J. Research 12, (1934) RP643.
} 
manometer. Such an error would actually never occur, since the measurements of saturation pressure in the thermometer do not magnify the effect of the impurity in the same manner as in the test for purity. Furthermore, had the impurity been hydrogen, almost all of it would have been discarded with the initial distillate and through the pump just prior to filling the saturation pressure thermometers (see following section of this paper). On the other hand, if the impurity had been nitrogen, it would not have been entirely discarded with the isolation of the initial distillate and a subsequent distillation of the condensate from the tube, $M$. The effect of the nitrogen should therefore be estimated. This may be done by assuming 0.00003 mole fraction of nitrogen in oxygen, and computing the saturation pressure of the mixture by means of Raoult's law. When this is done, it will be seen that this small amount of nitrogen will alter the saturation pressure of pure oxygen by $0.01 \mathrm{~mm}$. Since this represents a reproducibility not ordinarily attained in the measurement of the saturation pressures of oxygen in the region of its normal boiling point, the oxygen prepared may be considered satisfactory for the purpose intended.

\section{FINAL TREATMENT AND TRANSFER OF OXYGEN TO THE SATURATION PRESSURE THERMOMETER}

The initial distillate and the final residue obtained during the test for purity were discarded through the pump. The oxygen contained in the tube, $M$, was then distilled, a few milliliters being discarded through the pump. This distillation would effectively remove traces of hydrogen and some nitrogen. The test for purity did not indicate the necessity of this additional procedure, but it was performed as an additional precaution. Approximately $18 \mathrm{ml}$ of liquid oxygen then remained, and was isolated in the tube, $M$.

The graduated tube, $K$, and the saturation pressure manometer, $L$, were evacuated with the same care used in outgassing the apparatus prior to the preparation of the oxygen. The connections to $L$ were heated during this evacuation, and drastically flamed at the constriction where the thermometer was to be sealed off.

The technique used in filling the thermometer was designed to eliminate gases driven from the glass at the time the thermometer was sealed off. Oxygen was transferred from $M$ to $K$ by immersing the former in a bath of liquid oxygen and the latter in a bath of liquid air.

The amount collected in $K$ was $0.2 \mathrm{ml}$ (of liquid) in excess of the amount necessary to fill the thermometer to a pressure of 5 atmospheres (of gas) at room temperature. This oxygen was then condensed in the thermometer. The condensing bulb of the thermometer was now immersed in liquid oxygen, and the tube $K$, in liquid air. The flow of oxygen was consequently from the thermometer to the tube, $K$. While the excess of $0.2 \mathrm{ml}$ of liquid oxygen was being condensed back into $K$, with vapor flowing from the thermometer, the constriction joining the two was fused shut. Thus, gases driven from the fused glass were carried away from the thermometer.

Washington, August 25, 1938. 